\title{
Stability Analysis and Stabilization of Systems with Input Backlash
}

\author{
S. Tarbouriech, I. Queinnec, C. Prieur
}

\begin{abstract}
This paper deals with the stability analysis and stabilization of linear systems with backlash in the input. Uniform ultimate boundedness stability and stabilization problems are tackled allowing to characterize suitable regions of the state space in which the closedloop trajectories can be captured. In the state feedback control design, computational oriented solutions are derived to solve suboptimal convex optimization problems able to give a constructive solution.
\end{abstract}

Index Terms-Stability analysis, stabilization, backlash, uniform ultimate boundedness stability.

\section{INTRODUCTION}

Backlash operators are nonlinear elements with memory. They are usual phenomena in many physical systems, such as electrical inductors, piezo-actuators, gear trains and mechanical friction systems (see e.g. the survey [18] on such memory elements and also on other operators as Krasnolsel'skii-Pokrovskii hysteresis). Other closely related infinite dimensional operators are also considered in the literature such as the Preisach models [3], the Coleman-Hodgdon operator [7] and the counterclockwise input-output dynamics [1]. For many applications, such nonlinear operators affect the inputs of control systems (see e.g. [17], [19]). The negligence of such nonlinearities during the control design or the stability analysis can lead to an important degradation of the closed-loop performance or even to the loss of stability (see, in particular, [19]).

Here we focus on the stability analysis and the control design for a linear system with a backlash operator in the input. This induces to study an infinite dimensional system, since the backlash operator is a memory-based relation. Several approaches have been developed in the context of this type of non-smooth nonlinearity: see, for example [8] and references therein. Moreover, some solutions consisting in applying inverse nonlinearity have been proposed [25], [26]. However, although there is a large literature dealing with the stability analysis and the estimation of input/output properties (see e.g. [12], [20] for recent results), there are not many papers suggesting design methods for nonlinear systems with memory operators, except [13] where a dissipativity property is used, and [23] where the dynamics of the differential equation modeling a one-dimensional hysteresis is controlled.

In the present paper, sufficient conditions are provided to characterize a compact set for the nonlinear system as a finite-time attractor for all admissible initial conditions. In this set, the system trajectories are uniformly ultimately bounded. The method developed does not impose any assumption on the system matrix at $s=0$, where $s$ is the Laplace variable, as required in [21] and [22]. Furthermore, the approach pursued does not need to consider that the static gain of the system has to be either null or nonsingular contrary to [10] and [11]. More precisely, generalized sector conditions using properties of the backlash operator and Lyapunov arguments allow us to analyze the stability, i.e., we exhibit, in our first main result

S. Tarbouriech and I. Queinnec are with CNRS, LAAS, 7 avenue du colonel Roche, F-31400 Toulouse, France and Univ de Toulouse, LAAS, F-31400 Toulouse, France. tarbour@laas.fr, queinnec@laas.fr

C. Prieur is with Gipsa-lab, Department of Automatic Control, Grenoble Campus, 11 rue des Mathématiques, BP 46, 38402 Saint Martin d'Hères, France christophe.prieur@gipsa-lab.fr (see Theorem 2 below), some conditions to prove that a compact set is a finite-time attractor, for all admissible initial conditions. These conditions are given in terms of Linear Matrix Inequalities (LMI), which can be solved using standard numerical algorithms, within a time of computation which is polynomial with respect to the dimension of the data. Even if the spirit of the approach proposed is quite similar to the SISO statement in Corollary 16 in [12], it allows to deal with MIMO systems. Moreover, while both [12] and our paper address the characterization of the finite-time attractor, our approach, differently from [12], uses the knowledge of the Lyapunov function. In this sense, our approach can be considered as complementary with the method proposed in [12]. Furthermore, at the opposite of conditions developed in [24], we do not need to use the time-derivative version of the system. An implicit objective in the characterization of the finite-time attractor is to make it as small as possible. Then, the second contribution of the paper (see Theorem 3) resides in proposing a technique to design both the state feedback gain not only stabilizing the linear part of the system but also allowing to address the minimization of the associated finite-time attractor. In this sense, the conditions obtained are complementary to those resulting for the stability analysis. This design result is again based on Lyapunov techniques. The computational issues and several examples illustrating the interests and the drawbacks of the conditions are also presented.

Notation. For two vectors $x, y$ of $\Re^{n}$, the notation $x \succeq y$ means that $x_{(i)}-y_{(i)} \geq 0, \forall i=1, \ldots, n . \mathbf{1}$ and $\mathbf{0}$ denote the identity matrix and the null matrix of appropriate dimensions, respectively. $x \in \Re_{+}^{n}$ means that $x \succeq 0$. The Euclidian norm is denoted $\|\cdot\| . A^{\prime}$ and $\operatorname{trace}(A)$ denote the transpose and the trace of $A$, respectively. $H e\{A\}=A+A^{\prime} . \operatorname{diag}(A ; B)$ denotes the diagonal matrix which diagonal blocks are formed by squared matrices $A$ and $B$. For two symmetric matrices, $A$ and $B, A>B$ means that $A-B$ is positive definite. In partitioned symmetric matrices, the symbol $\star$ stands for symmetric blocks. $\lambda_{\max }(A)$ (respectively, $\left.\lambda_{\min }(A)\right)$ denotes the maximal (respectively, minimal) eigenvalue of the matrix $A$.

\section{PRoblem Formulation}

The class of systems under consideration is described by:

$$
\begin{aligned}
& \dot{x}=A x+B \Phi[w] \\
& w=K x
\end{aligned}
$$

where $x \in \Re^{n}$ is the state and $w \in \Re^{m}$ is the input of the nonlinearity $\Phi . A, B$ and $K$ are matrices of appropriate dimensions. The pair $(A, B)$ is supposed to be controllable. $K$ is the state feedback control gain to be designed or supposed known. $\Phi$ is a componentwise backlash operator (see, for example, [18], [21], [25], [3]). We denote the set of continuous, piecewise differentiable functions $f:[0,+\infty) \rightarrow \Re^{m}$ by $\mathcal{C}_{p w}^{1}\left([0,+\infty) ; \Re^{m}\right)$, that is the set of continuous functions $w$ being, for some unbounded sequence $\left(t_{j}\right)_{j=0}^{\infty}$ in $[0,+\infty)$ with $t_{0}=0$, continuously differentiable on $\left(t_{j-1}, t_{j}\right)$ for all $j \in \mathbb{N}$. Given the vector $\rho$ in $\Re_{+}^{m}$ and $L=\operatorname{diag}\left(\ell_{(i)}\right)$, with positive values $\ell_{(i)}, i=1, \ldots, m$, the operator $\Phi$ is defined as follows, for all $f \in \mathcal{C}_{p w}^{1}\left([0,+\infty) ; \Re^{m}\right)$, for all $j \in \mathbb{N}$, for all $t \in\left(t_{j-1}, t_{j}\right)$ and for all $i \in\{1, \ldots, m\}$ : 


$$
(\overbrace{\Phi[f]}(t))_{(i)}=\left\{\begin{array}{c}
\ell_{(i)} \dot{f}_{(i)}(t) \text { if } \dot{f}_{(i)}(t) \geq 0 \\
\text { and }(\Phi[f](t))_{(i)}=\ell_{(i)}\left(f_{(i)}(t)-\rho_{(i)}\right) \\
\ell_{(i)} \dot{f}_{(i)}(t) \text { if } \dot{f}_{(i)}(t) \leq 0 \\
\text { and }(\Phi[f](t))_{(i)}=\ell_{(i)}\left(f_{(i)}(t)+\rho_{(i)}\right) \\
0 \text { otherwise }
\end{array}\right.
$$

where $0=t_{0}<t_{1}<\ldots$ is a partition of $[0,+\infty)$ such that $f$ is continuously differentiable on each of the intervals $\left(t_{j-1}, t_{j}\right), j \in \mathbb{N}$. Thus, $\Phi$ is a time-invariant nonlinearity with slope restriction, as in [22]. Note however that it is a memory-based operator, since to compute it, we need to have information about the past values of $w$ (this is not the case in [22]). Such an operator can be depicted in Figure 1.

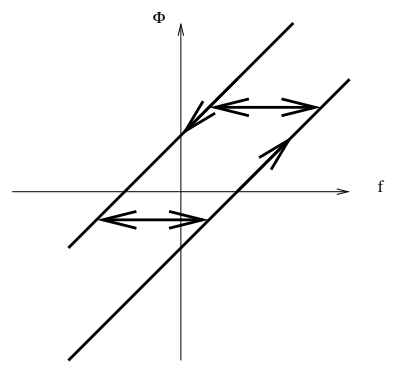

Fig. 1. An illustration of a backlash operator.

Since it is possible to stack several backlash operators into one backlash operator (with the dimension equal to the sum of all dimensions), without loss of generality, it is suitable to assume that only one backlash is present in (1).

Throughout the paper, we define the set of admissible initial conditions $(w(t=0))$, from which we want to guarantee the stability, as follows

$$
L(w(0)+\rho) \succeq \Phi[w](0) \succeq L(w(0)-\rho)
$$

According to [19], [8], that means that the nonlinearity $\Phi$ is active. Then, with (2), one gets

$$
L(w(t)+\rho) \succeq \Phi[w](t) \succeq L(w(t)-\rho), \forall t \geq 0
$$

In a neighborhood of the origin, system (1) operates in open loop and the presence of the backlash $\Phi[w](t)$ may induce the existence of multiple equilibrium points or a limit cycle. Thus, one aims at characterizing compact sets $\mathcal{S}_{0}$ that are globally attractive in finite-time, or equivalently, such that, for any initial condition $x(0)$ belonging to $\Re^{n} \backslash \mathcal{S}_{0}$, the resulting trajectory of the closed-loop system (1) is uniformly ultimately bounded in the set $\mathcal{S}_{0}$ (see [15, Section 4.8]). This is stated in the following problem:

Problem 1 (Stability analysis): Given a gain $K$ such that $A+$ $B L K$ is Hurwitz, characterize the region of the state space in which the solutions of system (1) are uniformly ultimately bounded when initialized as in (3).

Imposing $A+B L K$ Hurwitz is classical since it corresponds to system (1) when $\Phi[w]$ is neglected and replaced by $L w$. Let us emphasize that, unlike what is generally considered in the literature, in the current paper, the Hurwitz assumption on matrix $A$ has been relaxed, i.e. the matrix $A$ can be exponentially unstable.

The second problem we intend to solve is complementary to Problem 1. Actually, we want to make the domain $\mathcal{S}_{0}$ as small as possible, through the design of the state feedback gain $K$, as explicated below.

Problem 2 (Synthesis problem): Determine the gain $K$ such that $A+B L K$ is Hurwitz and which minimizes the size of the associated region of the state space in which the solutions of the system (1) are uniformly ultimately bounded when initialized as in (3).

\section{MAIN RESUlts}

\section{A. Preliminary results}

For conciseness, throughout the paper, we denote $\dot{\Phi}$ instead of $\overbrace{\Phi[w]}$, and $\Phi$ instead of $\Phi[w]$. Recall that the nonlinear operator $\Phi$ verifies the properties stated in Lemma 3.1 in [24]. Let us define the nonlinearity $\Psi$

$$
\Psi=\Phi-L w=\Phi-L K x
$$

Hence, from Lemma 3.1 in [24] and (4), we can formulate the following properties with respect to $\Psi$.

Lemma 1: For any diagonal positive definite matrices $N_{1}, N_{2}, N_{3}$ in $\Re^{m \times m}$, with $N_{3} \geq \mathbf{1}$, we have, for all $w \in \mathcal{C}_{p w}^{1}\left([0,+\infty) ; \Re^{m}\right)$, for all $t \in\left(t_{i-1}, t_{i}\right)$

$$
\begin{aligned}
&(\dot{\Psi}+L \dot{w})^{\prime} N_{1} \Psi \leq 0, \\
&(\dot{\Psi}+L \dot{w})^{\prime} N_{2}\left(\dot{\Psi}+\left(\mathbf{1}-N_{3}\right) L \dot{w}\right) \leq 0, \\
&-L \rho \preceq \Psi \preceq L \rho
\end{aligned}
$$

where $0=t_{0}<t_{1}<\ldots$ is a partition of $[0,+\infty)$ such that $w$ is continuously differentiable on each of the intervals $\left(t_{j-1}, t_{j}\right), j \in \mathbb{N}$.

Remark 1: Property (6) can be seen as a property of passivity with respect to the output $\dot{\Phi}$ and input $N_{1} L w$, and with the storage function $\frac{1}{2} \Phi^{\prime} N_{1} \Phi[15]$.

\section{B. Stability analysis}

The following result relative to Problem 1 can be stated.

Theorem 2: Given $K$ such that $A_{0}=A+B L K$ is Hurwitz. A positive scalar $\tau_{1}$ being given, if there exist a symmetric positive definite matrix $P \in \Re^{n \times n}$, three diagonal positive definite matrices $N_{1} \in \Re^{m \times m}, N_{2} \in \Re^{m \times m}$ and $T_{3} \in \Re^{m \times m}$ satisfying the following LMI conditions

$$
\left.\begin{array}{ccc}
H e\left\{A_{0}^{\prime} P\right\}+\tau_{1} P & \star & \star \\
B^{\prime} P-N_{1} L K A_{0} & -T_{3}-H e\left\{N_{1} L K B\right\} & \star \\
-N_{2} L K A_{0} & -N_{1}-N_{2} L K B & -2 N_{2}
\end{array}\right)<\mathbf{0}
$$

then, for any initial admissible conditions $(x(0), \Psi(0))$, the resulting trajectories of the closed-loop system (1) are uniformly ultimately bounded in the set $\mathcal{S}_{0}(P)$ defined as follows:

$$
\mathcal{S}_{0}(P)=\left\{x \in \Re^{n} ; x^{\prime} P x \leq 1\right\}
$$

Proof. Consider a quadratic Lyapunov function candidate $V$ defined by $V(x)=x^{\prime} P x, P=P^{\prime}>\mathbf{0}$, for all $x$ in $\Re^{n}$. We want to verify that there exists a class $\mathcal{K}$ function $\alpha$ such that $\dot{V}(x) \leq-\alpha(V(x))$, for all $x$ such that $x^{\prime} P x \geq 1$ (i.e. for any $x \in \Re^{n} \backslash \mathcal{S}_{0}$ ), and for all nonlinearities $\Psi$ satisfying Lemma 1 (i.e. satisfying relations (6), (7), (8)). By using the $\mathrm{S}$-procedure, it corresponds to verify that $\mathcal{L}<0$, where

$$
\begin{aligned}
\mathcal{L} & =\dot{V}(x)-\tau_{1}\left(1-x^{\prime} P x\right)-\sum_{i=1}^{m} \tau_{3 i}\left(\Psi_{(i)}^{\prime} \Psi_{(i)}-\rho_{(i)}^{2} \ell_{(i)}^{2}\right) \\
& -2(\dot{\Psi}+L \dot{w})^{\prime} N_{1} \Psi-2(\dot{\Psi}+L \dot{w})^{\prime} N_{2}\left(\dot{\Psi}+\left(\mathbf{1}-N_{3}\right) L \dot{w}\right)
\end{aligned}
$$

with $\tau_{1}$ a positive scalar and $T_{3}=\operatorname{diag}\left(\tau_{3 i}\right)$ a positive diagonal matrix. Choosing $N_{3}=1$ and noting that $\dot{w}=K A_{0} x+K B \Psi$ and $\dot{V}(x)=x^{\prime}\left(A^{\prime} P+P A\right) x+2 x^{\prime} P B \Phi$, or equivalently, from the 
definition of $\Psi$ in (5), $\dot{V}(x)=x^{\prime}\left(A_{0}^{\prime} P+P A_{0}\right) x+2 x^{\prime} P B \Psi$, it follows that $\mathcal{L}=\mathcal{L}_{0}+\rho^{\prime} L T_{3} L \rho-\tau_{1}$ with

$$
\mathcal{L}_{0}=\left(\begin{array}{c}
x \\
\Psi \\
\dot{\Psi}
\end{array}\right)^{\prime} \mathcal{M}_{0}\left(\begin{array}{c}
x \\
\Psi \\
\dot{\Psi}
\end{array}\right)
$$

where $\mathcal{M}_{0}$ is the left-hand-side matrix in (9). The satisfaction of relations (9) and (10) implies both $\mathcal{L}_{0}<0$ and $\rho^{\prime} L T_{3} L \rho-\tau_{1} \leq 0$, and then $\mathcal{L}<0$. Therefore, there exists $\varepsilon>0$, such that $\mathcal{L} \leq$ $-\varepsilon\left\|\left(x^{\prime} \Psi^{\prime} \dot{\Psi}^{\prime}\right)^{\prime}\right\|^{2} \leq-\varepsilon x^{\prime} x$. Hence, since by definition one gets $\dot{V}(x) \leq \dot{V}(x)-\tau_{1}\left(1-x^{\prime} P x\right) \leq \mathcal{L}$ one can also verify $\dot{V}(x)-$ $\tau_{1}\left(1-x^{\prime} P x\right) \leq-\varepsilon x^{\prime} x$, for any $x \in \Re^{n} \backslash \mathcal{S}_{0}$, or equivalently,

$$
\dot{V}(x) \leq-\varepsilon x^{\prime} x, \forall x \text { such that } x^{\prime} P x \geq 1 .
$$

Consider now a solution of (1) starting from an admissible initial condition at $t_{0}=0$ such that $x\left(t_{0}\right)^{\prime} P x\left(t_{0}\right) \geq 1$. According to (13), there exists a time $T \geq t_{0}+\left(x\left(t_{0}\right)^{\prime} P x\left(t_{0}\right)-1\right) \lambda_{\max }(P) / \epsilon$ such that $x(t) \in \mathcal{S}_{0}(P), \forall t \geq T$. Furthermore, $\mathcal{S}_{0}(P)$ is an invariant set for system (1). Hence, in accordance with [15], it follows that the trajectories are uniformly ultimately bounded in $\mathcal{S}_{0}(P)$ for any $x(0)$. That concludes the proof of Theorem 2 .

Theorem 2 ensures the finite-time convergence of the solutions of system $(1)$ inside $\mathcal{S}_{0}(P)$ for any initial admissible condition. Hence, the set $\mathcal{S}_{0}(P)$ contains the possible equilibrium points or limit cycles potentially induced by the backlash operator. The equilibrium points of system (1), when they exist, satisfy: $\dot{x}_{e}=\mathbf{0}=(A+B L K) x_{e}+$ $B \Psi_{e}$, or equivalently, since $A_{0}$ is Hurwitz, $x_{e}=-A_{0}^{-1} B \Psi_{e}$, $x_{e}^{\prime} P x_{e} \leq 1$ and $L \rho \succeq \Psi_{e} \succeq-L \rho$.

\section{Control design}

To address Problem 2 and to remove the products between $A_{0}$ and $K$ appearing in condition (9), the control design conditions are developed by using the Finsler lemma [2]. Such an approach is stated in the following result.

Theorem 3: A positive scalar $\tau_{1}$ being given, if there exist a symmetric positive definite matrix $P \in \Re^{n \times n}$, five matrices $Q_{1} \in \Re^{n \times n}$, $Q_{2} \in \Re^{n \times n}, F_{3} \in \Re^{m \times n}, F_{4} \in \Re^{m \times n}$ and $K \in \Re^{m \times n}$, three diagonal positive definite matrices $N_{1} \in \Re^{m \times m}, N_{2} \in \Re^{m \times m}$ and $T_{3} \in \Re^{m \times m}$ satisfying the matrix condition (14) (see top of the next page) and:

$$
\rho^{\prime} L T_{3} L \rho-\tau_{1} \leq 0
$$

then the state feedback gain $K$ is such that $A+B L K$ is Hurwitz and, for any initial admissible conditions $(x(0), \Psi(0))$, the resulting trajectories of the closed-loop system (1) are uniformly ultimately bounded in the set $\mathcal{S}_{0}(P)$ as defined in (11).

Proof. Consider the quadratic Lyapunov function defined by $V(x)=$ $x^{\prime} P x, P=P^{\prime}>\mathbf{0}$, for all $x$ in $\Re^{n}$. The time-derivative of $V(x)$ along the trajectories of system (1) reads $\dot{V}(x)=\dot{x}^{\prime} P x+x^{\prime} P \dot{x}$. Moreover, by noting $\xi=\left(\begin{array}{cccc}x^{\prime} & \dot{x}^{\prime} & \Psi^{\prime} & \dot{\Psi}^{\prime}\end{array}\right)^{\prime}$, system (1) can be also written as follows:

$$
\left(\begin{array}{llll}
A+B L K & \mathbf{- 1} & B & \mathbf{0}
\end{array}\right) \xi=\mathbf{0}
$$

We then want to verify that there exists a $\mathcal{K}$ function $\alpha$ such that $\dot{V}(x) \leq-\alpha(V(x))$, for all $x$ such that $x^{\prime} P x \geq 1$, for all nonlinearity $\Psi$ satisfying Lemma 1 and for $x, \Psi$ satisfying (16). In other words, by using the S-procedure, we can express $\mathcal{L}$ defined in (12) as $\mathcal{L}=$ $\xi^{\prime} \mathcal{L}_{0} \xi-\tau_{1}+\rho^{\prime} L T_{3} L \rho$, i.e., we want to verify:

$$
\xi^{\prime} \mathcal{L}_{0} \xi=\xi^{\prime}\left(\begin{array}{cccc}
\tau_{1} P & P & \mathbf{0} & \mathbf{0} \\
P & \mathbf{0} & -K^{\prime} L N_{1} & -K^{\prime} L N_{2} \\
\mathbf{0} & -N_{1} L K & -T_{3} & -N_{1} \\
\mathbf{0} & -N_{2} L K & -N_{1} & -2 N_{2}
\end{array}\right) \xi<0
$$$$
\text { and }-\tau_{1}+\rho^{\prime} L T_{3} L \rho \leq 0
$$

for $\xi$ such that $\left(\begin{array}{llll}A+B L K & \mathbf{- 1} & B & 0\end{array}\right) \xi=\mathbf{0}$

with $\tau_{1}$ a positive scalar and $T_{3}$ a positive diagonal matrix. By using the Finsler lemma, the satisfaction of (17) consists of finding some multipliers $F_{1} \in \Re^{n \times n}, F_{2} \in \Re^{n \times n}, F_{3} \in \Re^{m \times n}, F_{4} \in \Re^{m \times n}$ such that $\mathcal{L}_{0}+\operatorname{He}\left\{\mathcal{F}\left(\begin{array}{llll}A+B L K & \mathbf{- 1} & B & \mathbf{0}\end{array}\right)\right\}<\mathbf{0}$, with $\mathcal{F}=\left(\begin{array}{cccc}F_{1}^{\prime} & F_{2}^{\prime} & F_{3}^{\prime} & F_{4}^{\prime}\end{array}\right)^{\prime}$, which reads:

$$
\left(\begin{array}{ccc}
H e\left\{F_{1} A+F_{1} B L K\right\}+\tau_{1} P & \star & \\
P+F_{2} A+F_{2} B L K-F_{1}^{\prime} & -F_{2}-F_{2}^{\prime} \\
F_{3} A+F_{3} B L K+B^{\prime} F_{1}^{\prime} & -N_{1} L K+B^{\prime} F_{2}^{\prime}-F_{3} \\
F_{4} A+F_{4} B L K & -F_{4}-N_{2} L K & \\
& \star & \star \\
& \star & \star \\
& H e\left\{F_{3} B\right\}-T_{3} & \star \\
& -N_{1}+F_{4} B & -2 N_{2}
\end{array}\right)<\mathbf{0}
$$

A necessary condition for this inequality to be strict is the nonsingularity of matrix $F_{2}$. Hence, by considering that $F_{1}$ is also nonsingular and by multiplying the previous inequality at left by $\operatorname{diag}\left(Q_{1} ; Q_{2} ; \mathbf{1} ; \mathbf{1}\right)$ and at right by $\operatorname{diag}\left(Q_{1}^{\prime} ; Q_{2}^{\prime} ; \mathbf{1} ; \mathbf{1}\right)$ with $Q_{1}=$ $F_{1}^{-1}$ and $Q_{2}=F_{2}^{-1}$, one obtains relation (14). The satisfaction of relations (14) and (15) implies the satisfaction of condition (17). Furthermore, due to the block $(1,1)$ of the matrix in relation (14), the satisfaction of relation (14) means that the gain $K$ is such that $A+B L K$ is Hurwitz. Similarly to the proof of Theorem 2, by definition, $\mathcal{S}_{0}(P)$ is an invariant set for the trajectories of system (1). Hence, in accordance with [15], it follows that the trajectories are uniformly ultimately bounded in $\mathcal{S}_{0}(P)$ for any $x(0)$. That concludes the proof.

\section{Discussion on systems with backlash in the output}

The solution to the analysis problem (Theorem 2) may be directly extended to the case of backlash in the output, considering the following system:

$$
\left\{\begin{array} { l } 
{ \dot { x } = A x + B _ { 1 } u } \\
{ y = C x + D \Phi [ w ] }
\end{array} \quad \text { with } \left\{\begin{array}{l}
w=E x \\
u=K y
\end{array}\right.\right.
$$

which can also be written as:

$$
\dot{x}=\left(A+B_{1} K(C+D L E)\right) x+B_{1} K D \psi
$$

with $\psi=\Phi-L w$ defined as in equation (5). The static output feedback gain $K$ is supposed chosen such that $\left(A+B_{1} K(C+D L E)\right)$ is Hurwitz. Then, by denoting $A_{0}=\left(A+B_{1} K(C+D L E)\right)$ and $B=B_{1} K D$, Theorem 2 directly applies. It also applies to the case of a dynamic output feedback with backlash in the measured output. On the other hand, the extension of Theorem 3 to the design problem with backlash in the sensor is not direct.

\section{NUMERICAL IMPLEMENTATION}

\section{A. Computational issues}

As far as $\tau_{1}$ is fixed, conditions stated in Theorem 2 are linear in the decision variables. The problem may then be solved as an optimization problem to evaluate the smallest set $\mathcal{S}_{0}(P)$, typically described by its volume, proportional to $\sqrt{\operatorname{det}\left(P^{-1}\right)}$ [2], in which the trajectories of the closed-loop system are uniformly ultimately 


$$
\left(\begin{array}{cccc}
H e\left\{A Q_{1}^{\prime}+B L K Q_{1}^{\prime}\right\}+\tau_{1} Q_{1} P Q_{1}^{\prime} & \star & \star & \star \\
Q_{2} P Q_{1}^{\prime}+A Q_{1}^{\prime}+B L K Q_{1}^{\prime}-Q_{2} & -Q_{2}-Q_{2}^{\prime} & \star & \star \\
F_{3} A Q_{1}^{\prime}+F_{3} B L K Q_{1}^{\prime}+B^{\prime} & -N_{1} L K Q_{2}^{\prime}+B^{\prime}-F_{3} Q_{2}^{\prime} & F_{3} B+B^{\prime} F_{3}-T_{3} & \star \\
F_{4} A Q_{1}^{\prime}+F_{4} B L K Q_{1}^{\prime} & -F_{4} Q_{2}^{\prime}-N_{2} L K Q_{2}^{\prime} & -N_{1}+F_{4} B & -2 N_{2}
\end{array}\right)<\mathbf{0}
$$

bounded. In the numerical examples which follow, thanks to the use of Yalmip [16] to build the LMI optimization problem, we use the convex minimization problem to solve Problem 1:

$$
\begin{aligned}
& \text { min - geomean }(P) \\
& \text { under conditions }(9),(10)
\end{aligned}
$$

where geomean $(P)$ is the geometric mean of the eigenvalues of the matrix $P$.

To solve the design problem expressed through Theorem 3, we exploit the fact that matrices $Q_{1}, Q_{2}, F_{3}$ and $F_{4}$ are Finsler multipliers which may be considered as degrees of freedom in the design conditions. Actually, one selects $Q_{2}=Q_{1}, F_{3}=\mathbf{0}, F_{4}=\mathbf{0}$, and introduces the variables $Y=K Q_{1}^{\prime}, R=Q_{1} P Q_{1}^{\prime}, S_{1}=N_{1}^{-1}$ and $S_{2}=N_{2}^{-1}$. With this change of variables and by pre- and post-mulitplying the matrix in condition (14) by $\operatorname{diag}\left(\mathbf{1} ; \mathbf{1} ; S_{1} ; S_{2}\right)$, condition (14) is then updated as follows:

$$
\left(\begin{array}{cccc}
H e\left\{A Q_{1}^{\prime}+B L Y\right\}+\tau_{1} R & \star & \star & \star \\
R+A Q_{1}^{\prime}+B L Y-Q_{1} & -Q_{1}-Q_{1}^{\prime} & \star & \star \\
B^{\prime} & L Y+S_{1} B^{\prime} & -S_{1} T_{3} S_{1} & \star \\
\mathbf{0} & -L Y & -S_{2} & -2 S_{2}
\end{array}\right)<\mathbf{0}
$$

Then the control design Problem 2 is solved as the optimization problem:

$$
\begin{aligned}
& \min _{R, Y, Q_{1}, T_{3}, S_{2}} \alpha \\
& \text { under conditions }(15),(21),\left(\begin{array}{cc}
R & Q_{1} \\
Q_{1}^{\prime} & \alpha P_{g}^{-1}
\end{array}\right)>\mathbf{0}
\end{aligned}
$$

where

- $P_{g}$ is selected as the solution to the analysis problem (20) or as directions of interest for the minimization of the set $\mathcal{S}_{0}(P) \subseteq\left\{x \in \Re^{n}, x^{\prime} P_{g} x \leq \alpha\right\}$, with $P=\left(Q_{1}\right)^{-1} R\left(Q_{1}^{\prime}\right)^{-1}$, in which the trajectories of the closed-loop system are uniformly ultimately bounded;

- $\tau_{1}$ is selected, for example on a grid;

- $S_{1}$ is selected as the inverse of the solution $N_{1}$ to the analysis problem. A grid search may alternatively be performed to select $S_{1}$.

Additional constraints may be added to set the performance of the closed-loop system, such as a limit on the size of the gain, or a pole-placement requirement [6]. This can be done by prescribing additionally the following LMI condition with extra variable $R_{p p}$, issued from the use of Finsler Lemma, which allows to place the poles of $(A+B L K)$ in the disk centered in $\sigma$ with radius $r$ :

$$
\left(\begin{array}{cc}
-R_{p p} & Q_{1} \frac{\left(A^{\prime}-\sigma \mathbf{1}\right)}{r}+Y^{\prime} \frac{L^{\prime} B^{\prime}}{r} \\
\frac{(A-\sigma \mathbf{1})}{r} Q_{1}^{\prime}+\frac{B L}{r} Y & R_{p p}-Q_{1}-Q_{1}^{\prime}
\end{array}\right)>\mathbf{0}
$$

\section{B. Illustrative Examples}

1) Example 1: The first example is of academic nature and illustrates the conservatism of the approach. Consider the unstable system (1) defined by the following data:

$$
A=\left(\begin{array}{ll}
0 & 1 \\
0 & 0
\end{array}\right) ; \quad B=\left(\begin{array}{l}
0 \\
1
\end{array}\right) ; \quad \begin{gathered}
K=K_{a n}=\left(\begin{array}{c}
-2-3 \\
L=1 ; \rho=0.5
\end{array}\right) \\
L=1
\end{gathered}
$$

Considering $\tau_{1}=0.9$ and applying Theorem 2, the optimization problem (20) gives the solution to the stability analysis problem

$$
P_{a n}=\left(\begin{array}{cc}
15.9510 & 6.8038 \\
6.8038 & 15.1183
\end{array}\right) ; \quad \begin{aligned}
& N_{1 a n}=5.303310^{-5} \\
& N_{2 a n}=1.352510^{-4}
\end{aligned}
$$

for which the indicator of the volume is $v\left(P_{a n}\right)=\sqrt{\operatorname{det}\left(P_{a n}^{-1}\right)}=$ 0.0716. The influence of the parameter $\tau_{1}$ on the size of $\mathcal{S}_{0}(P)$ is illustrated in Figure 2 (the problem becomes unfeasible for $\tau_{1} \geq 2=$ $\left.2\left|\lambda_{\max }\left(A_{0}\right)\right|\right)$. An iterative search on $\tau_{1}$ allows to quickly select a convenient value for this parameter.

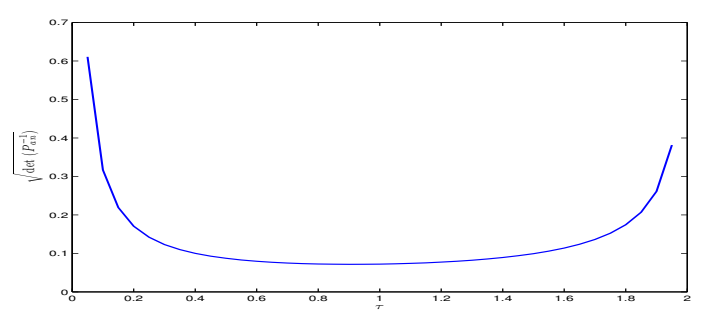

Fig. 2. Example 1 - Analysis problem. Influence of the parameter $\tau_{1}$ on the size of $\mathcal{S}_{0}(P)$.

For the synthesis problem, let us consider the optimization problem (22). $\tau_{1}$ is selected unchanged (equal to 0.9), $P_{g}=P_{a n}, S_{1}=N_{1 a n}^{-1}$. An additional pole-placement requirement (23) is used with $\sigma=-6$, $r=5$. A solution to the optimization problem (22) is given by

$$
K_{\text {syn }}=\left(\begin{array}{ll}
-10.3327 & -11.2293
\end{array}\right)
$$

It is recommended to proceed to the analysis of this new control gain $K_{\text {syn }}$, which results, for $\tau_{1}$ unchanged, to the solution $v\left(P_{\text {synan }}\right)=$ $\sqrt{\operatorname{det}\left(P_{\text {synan }}^{-1}\right)}=0.0057$, with

$$
P_{\text {synan }}=\left(\begin{array}{cc}
422.7379 & 33.4682 \\
33.4682 & 74.3510
\end{array}\right)
$$

The phase portraits of some trajectories of the system in closed loop with the control gain $K_{a n}$ (solid line) or $K_{\text {syn }}$ (dashed line), issued from the initial states $x(0)$, equal to $\left(\begin{array}{cc}0 & 1\end{array}\right)^{\prime}$ and $\left(\begin{array}{ll}1 & 1\end{array}\right)^{\prime}$, are plotted in Figure $3(\Phi[w](0)=L K x(0)$ which satisfies (3)). They show that the trajectories of the closed-loop system with $K_{a n}$ converge to a limit cycle included in the ellipsoid $\mathcal{S}_{0}\left(P_{a n}\right)$. The trajectories of the closed-loop system with $K_{s y n}$ are uniformly ultimately bounded in the set $\mathcal{S}_{0}\left(P_{\text {synan }}\right)$ included in the set $\mathcal{S}_{0}\left(P_{a n}\right)$. They may converge either to a limit cycle or to some equilibrium point (trajectory issued from $\left.\left(\begin{array}{ll}1 & 1\end{array}\right)^{\prime}\right)$, which are included in $\mathcal{S}_{0}\left(P_{\text {synan }}\right)$.

To evaluate the influence of the choice of $S_{1}$ on the solution to the design problem, a grid search in the sets defined by $S_{1}=$ $\left\{10^{3}, 10^{4}, 10^{5}, 10^{6}, 10^{7}\right\}, \tau_{1}=\{0.1,0.2, \cdots, 1.8,1.9\}$ has been performed. It gives as the best solution, after an additional analysis step $v\left(P_{\text {best }}\right)=\sqrt{\operatorname{det}\left(P_{\text {best }}^{-1}\right)}=0.0033$ (to be compared to the value 0.0057 obtained with a priori given values for $\tau_{1}$ and $S_{1}=N_{a n}^{-1}$ solutions to the analysis problem). 


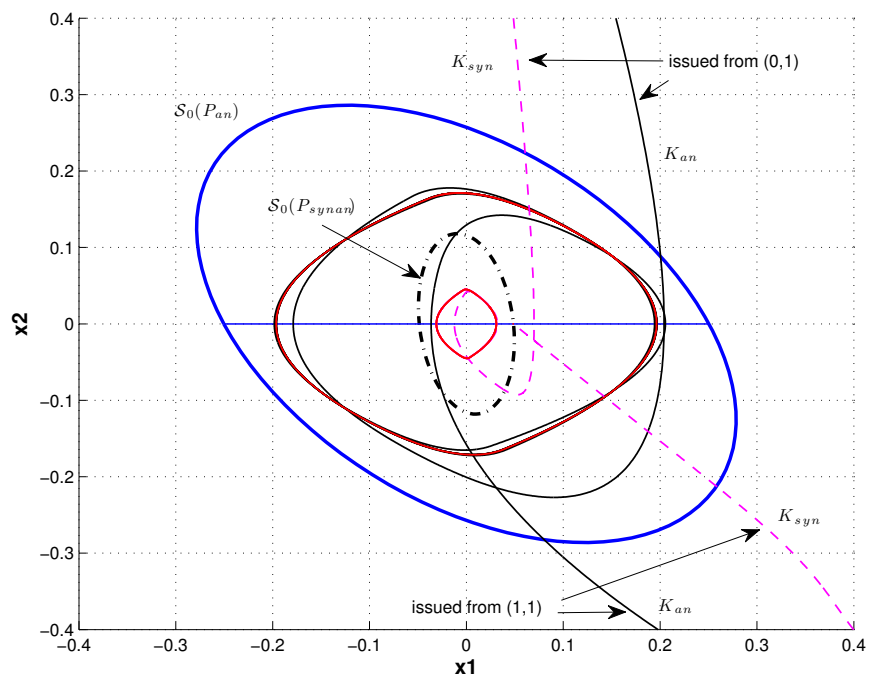

Fig. 3. Example 1. Ellipsoidal sets $\mathcal{S}_{0}\left(P_{a n}\right)$ and $\mathcal{S}_{0}\left(P_{\text {synan }}\right)$. Trajectories issued from $\left(\begin{array}{lll}0 & 1\end{array}\right)^{\prime}$ and $\left(\begin{array}{lll}1 & 1\end{array}\right)^{\prime}$ for the system closed with control gains $K_{a n}$ (solid line) and $K_{\text {syn }}$ (dashed line).

2) Example 2: As a second example, we consider an unstable F-8 aircraft MIMO example borrowed from [4], given by:

$A_{p}=\left(\begin{array}{cccc}-0.8 & -0.006 & -12 & 0 \\ 0 & -0.014 & -16.64 & -32.2 \\ 1 & -0.0001 & -1.5 & 0 \\ 1 & 0 & 0 & 0\end{array}\right) ; B_{p u}=\left(\begin{array}{cc}-19 & -3 \\ -0.66 & -0.5 \\ -0.16 & -0.5 \\ 0 & 0\end{array}\right)$

A backlash element interconnects the system with a state-feedback control, which values are given by

$$
\begin{gathered}
L=\left(\begin{array}{ll}
1 & 0 \\
0 & 1
\end{array}\right), \rho=\left(\begin{array}{l}
0.5 \\
0.5
\end{array}\right) \\
K=K_{a n}=\left(\begin{array}{cccc}
0.2672 & 0.0059 & -0.8323 & 0.8089 \\
0.5950 & -0.1534 & 2.1168 & 0.0525
\end{array}\right)
\end{gathered}
$$

Considering $\tau_{1}=1.5$ selected from an iterative procedure, the optimization problem (20) gives the solution $v\left(P_{a n}\right)=\sqrt{\operatorname{det}\left(P_{a n}^{-1}\right)}=$ 17.7848, with

$$
P_{a n}=\left(\begin{array}{cccc}
0.1381 & 0.0035 & -0.2112 & 0.2818 \\
0.0035 & 0.0266 & -0.6522 & 0.4999 \\
-0.2112 & -0.6522 & 16.7322 & -13.0185 \\
0.2818 & 0.4999 & -13.0185 & 11.5846
\end{array}\right)
$$

For the synthesis case, one considers $P_{g}=P_{a n}$ and a poleplacement requirement with $\sigma=-10, r=8.5$. A grid search in the sets defined by $S_{1}=\left\{10^{2} \mathbf{1}, 10^{3} \mathbf{1}, 10^{4} \mathbf{1}, 10^{5} \mathbf{1}, 10^{6} \mathbf{1}\right\}$, $\tau_{1}=\{1.1,1.2, \cdots, 2.1,2.2\}$, followed by an additional analysis step, allows to determine the control gain

$$
K_{\text {syn }}=\left(\begin{array}{cccc}
0.9533 & 0.2757 & -6.6111 & 7.9905 \\
0.1246 & -2.0493 & 37.6241 & -24.2286
\end{array}\right)
$$

with associated set defined from $P_{\text {synan }}$ with indication of the volume $v\left(P_{\text {synan }}\right)=\sqrt{\operatorname{det}\left(P_{\text {synan }}^{-1}\right)}=0.0831$. The time-evolution of the state $x$ is plotted in Figure 4, issued from the initial state $x(0)=\left(\begin{array}{cccc}1 & 1 & 1 & 1\end{array}\right)^{\prime}$ and initial nonlinearity $\Phi[w](0)=$ $L K x(0)$. It brings out the reduction of the size of the limit cycle with the gain $K_{\text {syn }}$ (plots in dashed line) in accordance with the objective which was actually to reduce the set $\mathcal{S}_{0}(P)$. Note however that this induces larger amplitude of the system input $\Phi[w]$, as it may also be seen on the form of the backlash operator characteristic in Figure 5.
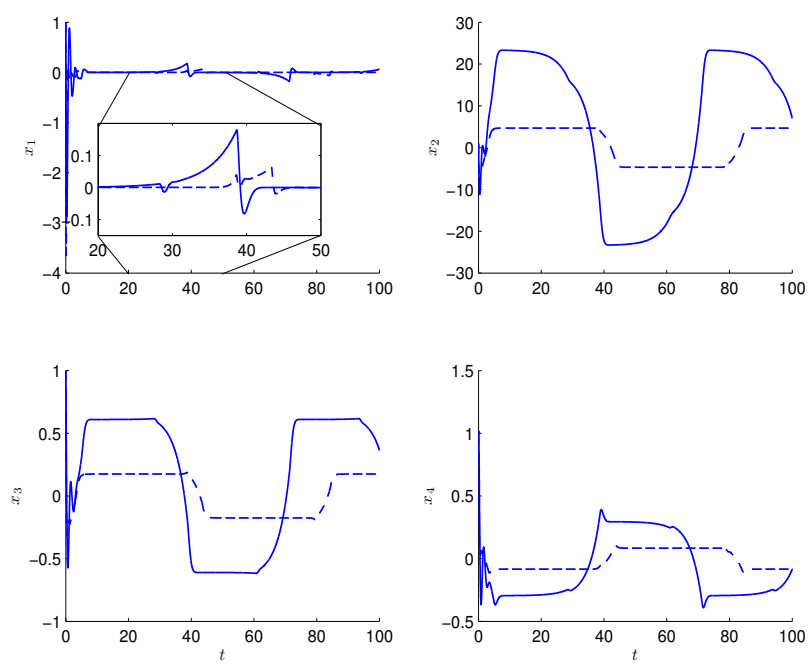

Fig. 4. Example 2 - Time-evolution of the state $x$. System in closed loop with $K_{a n}$ (solid line) or $K_{\text {syn }}$ (dashed line) + zoom of the steady-state behavior of $x_{1}$ between $t=20$ and $t=50$.
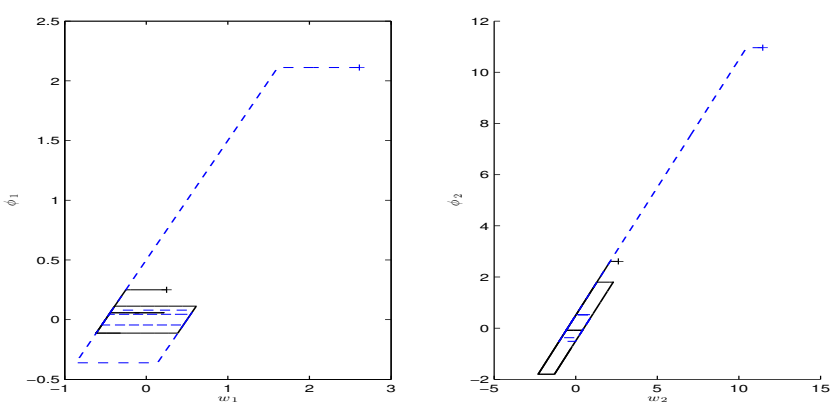

Fig. 5. Example 2 - Backlash characteristic $\Phi[w]$. System in closed loop with $K_{a n}$ (solid line) or $K_{s y n}$ (dashed line).

To evaluate the degree of conservatism of the sets $\mathcal{S}_{0}\left(P_{a n}\right)$ and $\mathcal{S}_{0}\left(P_{\text {synan }}\right)$ in which the trajectories are uniformly ultimately bounded, the time-evolution of $x^{\prime} P_{a n} x$ (solid line, associated to the control gain $K_{a n}$ ) and $x^{\prime} P_{\text {synan }} x$ (dashed line, associated to the control gain $K_{s y n}$ ) are plotted in Figure 6 . They show that, once the trajectory enters the ellipsoidal set $\mathcal{S}_{0}(P)$, i.e. $x^{\prime} P x \leq 1, P=P_{a n}$ or $P=P_{\text {synan }}$, it evolves over the time and comes more or less close to the boundary but never exits.

3) Example 3: A third example is proposed to illustrate how the analysis conditions stated in Theorem 2 may be used in the case of a feedback between the system output and the backlash input involving a dynamic system. This is typically the case when considering a dynamic feedback controller or an actuator device system such as proposed in [9], defined by:

$\left\{\begin{array}{l}\dot{x}_{1}=x_{2} \\ \dot{x}_{2}=c_{1} x_{1}+c_{2} x_{2}+\Phi[w]\end{array}\right.$ with $\left\{\begin{array}{l}\dot{x}_{A}=c_{3} x_{A}+v \\ w=x_{A}\end{array}\right.$

and a backlash operator with $L=1, \rho=1.5$. For numerical evaluation, values slightly different from [9] are used to manipulate an unstable open-loop system. We consider the coefficients $c_{1}=1$, $c_{2}=-1, c_{3}=-5$ and a state feedback control $v=-9 x_{1}-3 x_{2}$. 


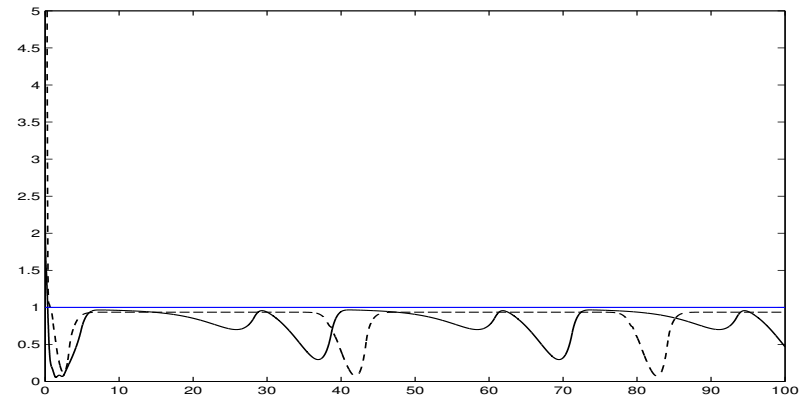

Fig. 6. Example 2 - Time-evolution of $x^{\prime} P_{a n} x$ (solid line) associated to the control gain $K_{a n}$ and of $x^{\prime} P_{\text {synan }} x$ (dashed line) associated to the control gain $K_{\text {syn }}$.

The structure of system (1) is retrieved with

$A=\left(\begin{array}{ccc}0 & 1 & 0 \\ 1 & -1 & 0 \\ -9 & -3 & -5\end{array}\right) ; B=\left(\begin{array}{l}0 \\ 1 \\ 0\end{array}\right) ; \quad K=\left(\begin{array}{ccc}0 & 0 & 1\end{array}\right)$

Using the optimization procedure (20) and an iterative search of $\tau_{1}$, one obtains, for $\tau_{1}=0.65$,

$$
P_{a n}=\left(\begin{array}{ccc}
150.9329 & 19.7677 & 82.7974 \\
19.7677 & 2.9032 & 10.7988 \\
82.7974 & 10.7988 & 45.4846
\end{array}\right)
$$

The phase portrait and backlash characteristic of the trajectory issued from ( $\left.\begin{array}{ccc}5 & 5 & 5\end{array}\right)^{\prime}$ are depicted in Figures 7-a and 7-b, respectively.

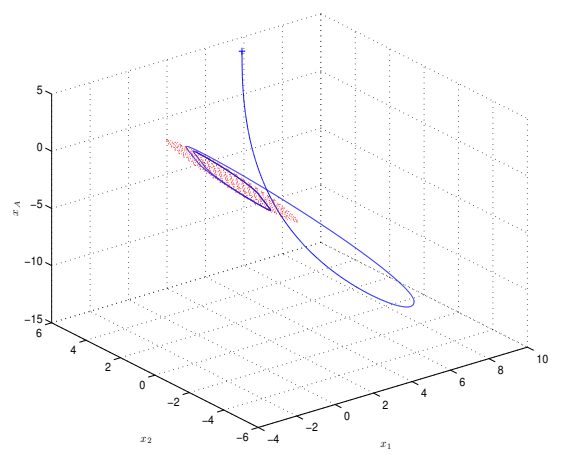

a

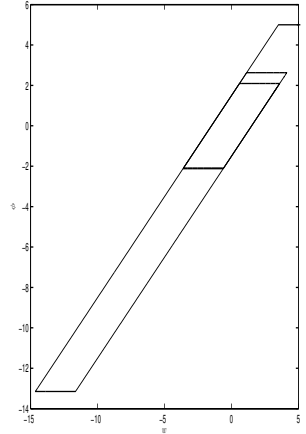

b
Fig. 7. Example 3 - a. Ellipsoidal set $\mathcal{S}_{0}\left(P_{a n}\right)$ and trajectory issued from $\left(\begin{array}{l}5 \\ 5\end{array} 5\right)^{\prime}$ - b. Backlash characteristic $\Phi[w]$.

\section{CONCLUDING REMARKS}

This paper considered systems with backlash operator in the input, aiming at analyzing the stability of such control systems. Uniform ultimate boundedness stability and stabilization problems were tackled allowing to characterize suitable regions of the state space in which the closed-loop trajectories can be captured. Future research directions are open. One idea would be to extend the technique to more general models of nonlinearities as studied, for example, in [5], [14]. Furthermore, as recalled in [12, Page 38], any Prandtl operator may be derived from an infinite weighted sum of backlash operators. It may be fruitful to adapt the present Lyapunov technic to such nonlinear systems. It may also be interesting to consider set-valued nonlinear operators encompassing Prandtl operators (as in [12, Page 37]). Finally, the case of a feedback between the system output and the backlash input involving an actuator device system subject to other nonlinearities (like, for example, saturation) should be considered.

\section{REFERENCES}

[1] D. Angeli. Systems with counterclockwise input-output dynamics. IEEE Transactions on Automatic Control, 51(7):1130-1143, 2006.

[2] S. Boyd, L. El Ghaoui, E. Feron, and V. Balakrishnan. Linear Matrix Inequalities in System and Control Theory. SIAM Studies in Applied Mathematics, 1994.

[3] M. Brokate and J. Sprekels. Hysteresis and phase transitions, volume 121 of Applied Mathematical Sciences. Springer-Verlag, New York, 1996.

[4] E.B. Castelan, S. Tarbouriech, J.M. Gomes da Silva Jr., and I. Queinnec. $\mathcal{L}_{2}$-stabilization of continuous-time systems with saturating actuators. International Journal of Robust and Nonlinear Control, 16:935-944, 2006.

[5] V. Cerone and D. Regruto. Bounding the parameters of linear systems with input backlash. IEEE Transactions on Automatic Control, 52(3):531-536, 2007.

[6] M. Chilali and P. Gahinet. $H_{\infty}$ design with pole placement constraints: an LMI approach. IEEE Transactions on Automatic Control, 41(3), 1996

[7] B.D. Coleman and M.L. Hodgdon. A constitutive relation for rateindependent hysteresis in ferromagnetically soft materials. International Journal of Engineering Science, 24(6):897-919, 1986.

[8] M. L. Corradini and G. Orlando. Robust stabilization of nonlinear uncertain plants with backlash or dead zone in the actuator. IEEE Transactions on Control Systems Technology, 10(1):158 - 166, 2002.

[9] M. L. Corradini, G. Orlando, and G. Parlangeli. Variable structure control of systems with sandwiched backlash. In 44th IEEE Conference on Decision and Control (CDC), pages 8112-8116, Sevilla, Spain, 2005.

[10] W.M. Haddad, V.-S. Chellaboina, and J. Oh. Linear controller analysis and design for systems with input hystereses nonlinearities. Journal of the Franklin Institute, 340:371-390, 2003.

[11] W.M. Haddad and V. Kapila. Absolute stability criteria for multiple slope-restricted monotonic nonlinearities. IEEE Transactions on Automatic Control, 40(2):361-365, 1995.

[12] B. Jayawardhana, H. Logemann, and E.P. Ryan. The circle criterion and input-to-state stability. IEEE Control Systems Magazine, 31(4):32-67, 2011.

[13] B. Jayawardhana, R. Ouyang, and V. Andrieu. Stability of systems with Duhem hysteresis operator: Dissipativity approach. Systems \& Control Letters, 162(3):286-293, 2013.

[14] B. Jayawarshana and V. Andrieu. Sufficient conditions for dissipativity on Duhem hysteresis model. In IEEE Conference on Decision and Control, pages 4378-4383, Shanghai, China, December 2009.

[15] H.K. Khalil. Nonlinear Systems. Prentice-Hall, 3rd edition, 2002.

[16] J. Löfberg. Yalmip: A toolbox for modeling and optimization in matlab. In Proceedings of the CACSD Conference, Taipei, Taiwan, 2004.

[17] H. Logemann and A. D. Mawby. Discrete-time and sampled-data lowgain control of infinite-dimensional linear systems in the presence of input hysteresis. SIAM Journal on Control Optimization, 41(1):113$140,2002$.

[18] J. W. Macki, P. Nistri, and P. Zecca. Mathematical models for hysteresis. SIAM Review, 35(1):94-123, 1993.

[19] M. Nordin, X. Ma, and P.O. Gutman. Controlling mechanical systems with backlash: a survey. Automatica, 38:1633-1649, 2002.

[20] R. Ouyang and B. Jayawardhana. Stability analysis and controller design for a system with hysteresis. In Proc. IEEE American Control Conference, Montreal, Canada, 2012.

[21] T. Paré, A. Hassibi, and J. How. A KYP lemma and invariance principle for systems with multiple hysteresis non-linearities. International Journal of Control, 74(11):1140-1157, 2001.

[22] P.G. Park, D. Banjerdpongchai, and T. Kailath. The asymptotic stability of nonlinear (Lur'e) systems with multiple slope restrictions. IEEE Transactions on Automatic Control, 43(7):979-982, 1998.

[23] C.-Y. Su, Y. Stepanenko, J. Svoboda, and T.P. Leung. Robust adaptive control of a class of nonlinear systems with unknown backlash-like hysteresis. IEEE Transactions on Automatic Control, 45(12):2427-2432, 2000.

[24] S. Tarbouriech, C. Prieur, and I. Queinnec. Stability analysis for linear systems with input backlash through sufficient LMI conditions. Automatica, 46(11):1911-1915, 2010.

[25] A. Taware and G. Tao. Control of sandwich nonlinear systems. Lecture Notes in Control and Information Sciences, vol.288, Springer-Verlag, Berlin, 2003.

[26] A. Taware, G. Tao, and C. Teolis. Design and analysis of a hybrid control scheme for sandwich nonsmooth nonlinear systems. IEEE Transactions on Automatic Control, 47(1):145-150, 2002. 